\title{
Der soziale Rechtsstaat im Widerstreit
}

Zur Verfassungsinterpretation Wolfgang Abendroths aus Anlass seines Ioo. Geburtstags

Die zentrale Norm des Grundgesetzes, dass die Bundesrepublik ein demokratischer und sozialer Rechtsstaat ist, dessen Gewährleistungsfunktion selbst durch die verfassungsändernden Gesetzgeber nicht eingeschränkt oder aufgehoben werden kann (Art. 20 Abs. I, Art. 79 Abs. 3 GG), wird von Wolfgang Abendroth 1953/54 bereits unter defensiven Bedingungen interpretiert, durch die er in der Staatsrechtslehre von Anfang an nur eine Minderheitsposition einnahm. Obgleich er Mitglied des Vorstands der Vereinigung Deutscher Staatsrechtslehrer war, ausgewiesen durch eine Fülle verfassungs- und völkerrechtlicher Arbeiten, beschloss der Vorstand mehrheitlich, Abendroth keines der beiden Hauptreferate für die Tagung der Staatsrechtslehrer von 1953 zu übertragen. ${ }^{.}$Die einzige Konzession war, dass Abendroth in der Aussprache den ersten Diskussionsbeitrag halten durfte. Mit einem Hauptreferat wurde - Personalentscheidungen sind auch immer Entscheidungen für eine bestimmte Sachposition - Ernst Forsthoff betraut, der 1933 in seiner Schrift »Der totale Staat $\aleph^{2}$ die Beseitigung der demokratischen Verfassung von Weimar, die Zerschlagung der Arbeiterbewegung und die Entrechtung der Juden legitimiert hatte. Die Reintegration der einstigen juristischen Trägerschichten der NS-Diktatur, vermittelt durch das I3 Ier Gesetz von I95 I, war schon fast vollständig abgeschlossen. Das hatte zur Folge, dass die Interpretationsherrschaft über das Grundgesetz, sieht man vom Bundesverfassungsgericht $a b$, in weitem Maße in den Händen der früheren juristischen Legitimationshelfer des Naziregimes lag. Dadurch konnte sich eine Tendenz zur Umdeutung zentraler Verfassungspositionen des Grundgesetzes - von der Entlegitimierung des Demonstrationsstreiks über die Ausweitung der privaten Eigentumsgarantie bis zur Entgrenzung des Begriffs der Kampfmaßnahme - ausbilden,3 die ihren Niederschlag auch in der Auslegung der Sozialstaatsklausel durch Forsthoff fand.

Die strategische Linie von Forsthoff bestand darin, die Prinzipien des sozialen Rechtsstaats zugunsten des isoliert gefassten liberalen Rechtsstaatsbegriffes weitgehend ins Leere laufen zu lassen. Forsthoff, der 1933 an der Universität Frankfurt am Main den Lehrstuhl von Hermann Heller nach dessen Ausschaltung durch die Nationalsozialisten übernimmt ${ }^{4}$ und fast mit innerer Konsequenz darauf verzichtet, die zentrale Studie Hellers zum Begriff des sozialen Rechtsstaats von 1929 zu zitieren, sprach dem Wort sozial jede verfassungsrechtliche Bedeutung ab. Forsthoff tilgte durch seine Exegese den Begriff des sozialen Rechtsstaats aus dem Grundgesetz. Er erklärte, dass diese Verfassungsbestimmung, im Gegensatz zu seinem Postulat, das Verfassungsgesetz positivistisch ernst zu nehmen, keine rechtliche Bedeutung habe: »Die Formel ssozialer

\footnotetext{
I Wolfgang Abendroth, Ein Leben in der Arbeiterbewegung, Frankfurt/Main 1976, S. 2 I 3.

2 Ernst Forsthoff, Der totale Staat, Hamburg 1933.

3 Joachim Perels, Das juristische Erbe des »Dritten Reiches «, Frankfurt/Main 1996, S. I 2 I ff., S. 29 f.; Wolfgang Abendroth, Innergewerkschaftliche Willensbildung, Urabstimmung und >Kampfmaßnahme<, in: ders., Antagonistische Gesellschaft und politische Demokratie, Neuwied I 972, S. 25 I ff.

4 Michael Stolleis, Geschichte des öffentlichen Rechts in Deutschland. Dritter Band I9I4-I945, München I999, S. 266; Klaus Meyer, Hermann Heller. Eine biographische Skizze, in: Christoph Müller u. a. (Hrsg.), Der soziale Rechtsstaat, Baden-Baden 1984, S. 84.
} 
Rechtsstaat< ist kein Rechtsbegriff «.5 Das heißt für Forsthoff: »Das sozialstaatliche Bekenntnis ... berührt die strukturelle Verfassungsform der Bundesrepublik nicht «. ${ }^{6}$ Diese anti-verfassungsrechtlichen Setzungen, die schon den Wortlaut des Grundgesetzes zum Verschwinden bringen, beruhen darauf, dass Forsthoff mit Hilfe eines klassisch bürgerlichen Rechtsstaatsbegriffs die vorhandene Gesellschaftsordnung aus der Verfügungsgewalt des demokratischen Staats ausgliedert: »Die Entscheidung für das soziale Element wird notwendig dazu führen, dass das Grundgesetz seine Gewährleistungsfunktion nur im Rahmen dessen ausübt, was von der jeweiligen Mehrheit und ihrer Regierung als sozial verstanden wird ... In diesem Fall wäre die Formel sozialer Rechtsstaat ein Mittel für unübersehbare Diskriminierungen und Verwirkungen. Das wäre aber die Vernichtung des Rechtsstaats. «7 Was Forsthoff polemisch »Verwirkungen und Diskriminierungen " nennt, sind die vom Grundgesetz selber festgelegten Bestimmungsmöglichkeiten über die Eigentumsordnung, die verfassungsrechtlich nicht auf deren private bürgerliche Formbestimmtheit reduziert ist (Art. I4 Abs. I Satz 2, Art. Is GG). Die Überhöhung des bürgerlichen Rechtsstaatsmoments mit dem Ziel, die Verpflichtungskraft der Sozialstaatsklausel auszuhöhlen, ist keine Verfassungsauslegung, sondern eine zur Umdeutung des Grundgesetzes eingeführte politische Option.

Abendroths Interpretation des Begriffs des sozialen Rechtsstaats ist der umfassende Versuch, den Normgehalt einer verfassungsgestaltenden Grundentscheidung im Blick auf die Möglichkeit einer legalen Veränderung der kapitalistischen Gesellschaft ohne jede Abstriche festzuhalten. Hierfür wird der Blick auf den Entstehungszusammenhang des Gedankens des sozialen Rechtsstaats gerichtet. Abendroth bezieht sich im direkten Gegensatz zu Forsthoff auf Hellers programmatische Schrift »Rechtsstaat oder Diktatur « aus der Endphase von Weimar, deren Perspektive in einer Vergesetzlichung der Wirtschaft ${ }^{8}$ durch die Schaffung eines sozialen Rechtsstaats besteht. Er soll insbesondere als Schutzwall gegen die Überwältigung der parlamentarischen Demokratie durch die wesentlichen Gruppen des Industriebürgertums fungieren. Diese politische Intention war für jene Kräfte im Parlamentarischen Rat bestimmend, die gesellschaftliche Lehren aus der Zerstörung der Weimarer Demokratie ziehen wollten. Für sie war der soziale Rechtsstaat die grundlegende Antwort auf die zur Sicherung des Kapitalismus errichtete Diktatur des Dritten Reiches. Daher ist es nicht zufällig, dass - Abendroth selber weist darauf hin - die Einfügung des Rechtsbegriffs des sozialen Rechtsstaats in das Grundgesetz auf den verfassungspolitischen Sprecher der SPD im Parlamentarischen Rat, auf Carlo Schmid zurückgeht. 9

Der Sozialstaatsgedanke hat für Abendroth eine doppelte Bedeutung. Zum einen bezeichnet er ein von den politischen Kräften im Parlamentarischen Rat gemeinsam vertretenes Minimum von sozialen Gerechtigkeitsvorstellungen, wie sie

5 Ernst Forsthoff, Begriff und Wesen des sozialen Rechtsstaats (I953), in: ders. (Hrsg.), Rechtsstaatlichkeit und Sozialstaatlichkeit, Darmstadt 1968, S. i88; Ernst Forsthoff, Die Umbildung des Verfassungsgesetzes, in: ders., Rechtsstaat im Wandel, München 1976, S. I3o ff.

6 Ebd., S. I91.

7 Ebd., S. I 87.

8 Hermann Heller, Rechtsstaat oder Diktatur (1929), in: ders., Gesammelte Schriften Bd. II, Tübingen I992, S. $46 \mathrm{I}$.

9 Wolfgang Abendroth, Zum Begriff des demokratischen und sozialen Rechtsstaats im Grundgesetz der Bundesrepublik Deutschland (I954), in: ders. (Fn. 3), S. iog ff., S. I I 2; zur Entwicklung des Denkens von Abendroth vgl. Abendroth, Gesammelte Schriften, Bd. I, I926-1948, hrsg. v. Michael Buckmiller/ Uli Schröder/Joachim Perels, Hannover 2006. 
etwa im Bundessozialhilfegesetz von 196I mit dem Postulat der Wahrung der Würde des Menschen Ausdruck finden.

Zum anderen zielt der Begriff des sozialen Rechtsstaats auf eine Umkehrung des Verhältnisses von Staat und Gesellschaft. Das System sozialer Ungleichheit wird nicht mehr als vorgegebene, gleichsam naturhafte Basis des Staats, die dessen Bestimmungsgewalt in Form eines lediglich liberalen Rechtsstaats vollständig entzogen ist, verfassungsrechtlich festgeschrieben: "Das entscheidende Moment des Gedankens der Sozialstaatlichkeit ... besteht ... darin, dass der Glaube an die immanente Gerechtigkeit der bestehenden Wirtschafts- und Gesellschaftsordnung aufgehoben ist und dass deshalb die Wirtschafts- und Gesellschaftsordnung der Gestaltung durch diejenigen Staatsorgane unterworfen wird, in denen sich die demokratische Selbstbestimmung des Volkes repräsentiert. «10 Allerdings hält Abendroth in genauer Anlehnung an die mit dem Sozialstaatsgedanken zusammenhängenden Grundrechte der Eigentumsgarantie, deren Ausgestaltung dem demokratischen Gesetzgeber überantwortet ist (Art. I4 Abs. I Satz 2 GG) und des Sozialisierungsartikels (Art. Is GG), der es zulässt, die großen privaten Produktionsstätten und Finanzinstitute in öffentliches Eigentum zu überführen, daran fest, dass sich aus dem Grundgesetz nicht der gleichsam einklagbare, verfassungsrechtlich normierte Anspruch ergibt, die privatwirtschaftliche Ordnung zu überwinden. Der Rechtsgrundsatz des sozialen Rechtsstaats gebietet dies nicht, aber er ermöglicht es in dem Sinne, dass der Schaffung einer gemeinwirtschaftlichen Ordnung keine verfassungsrechtlichen Schranken entgegenstehen.

Abendroths Interpretation des Sozialstaatsgedankens, die sich zu Beginn der 50er Jahre mit bestimmten programmatischen Festlegungen der Gewerkschaften und auch der SPD berührte, hatte zwar einige Auswirkungen in Kommentierungen des Grundgesetzes, etwa von Helmut Ridder und Michael Kittner. ${ }^{\text {II }}$ Auch in der Rechtsprechung des Bundesverfassungsgerichts zur wirtschaftspolitischen Neutralität des Grundgesetzes und zu egalitären, dem Teilhabegedanken entsprechenden Zugangsregelungen zur Universität wird Abendroths Sichtweise des Rechtsgrundsatzes des sozialen Rechtsstaats - als einer Norm, die die Erweiterung sozialer Gleichheit ermöglicht - in gewissem Sinne wirksam. ${ }^{\text {2 }}$

In den meisten Kommentaren jedoch, in denen sich der mainstream des öffentlichen Rechts niederschlägt, wird diese Ausprägung des Sozialstaatsgedankens, wie sie in der Tradition der demokratischen Arbeiterbewegung in Deutschland und ihrer Repräsentanten im Parlamentarischen Rat entwickelt worden ist, mehr oder minder offen negiert. Roman Herzog spricht in seiner Kommentierung von I 980 davon, dass das Sozialstaatsprinzip »zu einseitiger und überspitzter Interpretation aus ideologischer Voreingenommenheit verleitet«. Dem auf die Einschränkung und Transformation privater gesellschaftlicher Hierarchien bezogenen Gedanken demokratischer Teilhabe, den Abendroth in den Mittelpunkt seiner Interpretation stellt, spricht Herzog die verfassungsrechtliche Begründbarkeit ausdrücklich ab: »Die Unzulänglichkeiten der heutigen Gesellschaft ... dürfen unter der Verfassungsordnung des Grundgesetzes nicht durch eine egalitäre Gesellschaft ersetzt werden, in der die Freiheit des Menschen überhaupt 
nichts oder wenig gilt.«13 Herzog unterliegt dem Irrtum, »als ob eine gesellschaftliche Ordnung, die die Teilhabemöglichkeiten am sozialen Lebensprozess durch Formen der Mitbestimmung und der Selbstbestimmung erweitert, die Geltung des Freiheitsprinzips unterminiert. Mit diesem Argument, das für eine in der Sowjetunion unter Stalin charakteristische, despotisch strukturierte Gesellschaft mit Produktionsmitteln in Staatseigentum zutreffend ist, kann jedoch die ursprüngliche, von Karl Marx über Eduard Bernstein bis Rosa Luxemburg und Wolfgang Abendroth vertretene Konzeption eines Sozialismus in Form der Selbstbestimmung der Produzenten nicht widerlegt werden. ${ }^{14}$ Herzog geht auf diese demokratische Argumentationslinie in der Theorie der Arbeiterbewegung, die auch in Hermann Hellers Begriff des sozialen Rechtsstaats ihren Niederschlag findet, gar nicht ein. Auch die historischen Versuchsgestalten einer nicht mehr privaten bzw. autoritär-staatlichen Organisation und Lenkung des Produktionsprozesses, die sich im Prager Frühling von 1968 oder im chilenischen Transformationsmodell unter Allende zu Beginn der 7oer Jahre ausgebildet hatten, ${ }^{15}$ werden keines theoretischen Blicks gewürdigt, obgleich an jenen Prozessen die These, dass Bedingungen größerer gesellschaftlicher Gleichheit in Gestalt demokratischer Teilhabeformen zu einer Beschränkung, gar Aufhebung individueller Freiheit führten, grundlegend in Frage gestellt werden könnte.

In einem späteren, inzwischen einflussreichen Grundgesetzkommentar von I 998 wird einem wichtigen Element des Rechtsgrundsatzes des sozialen Rechtsstaats auf andere Weise das Gewicht genommen. Rolf Gröschner wendet sich dagegen, Hermann Heller und Carlo Schmid, den Sprecher der Sozialdemokratie im Parlamentarischen Rat, zu den maßgebenden gedanklichen Vätern des Sozialstaatsgrundsatzes der Verfassung zu machen, denn der Parlamentarische Rat habe sich "weder auf eine theoretische Konzeption noch auf ein politisches Programm des Sozialstaats festlegen wollen ${ }_{.}{ }^{16}$ Durch diese These, die ihr relatives Recht hat, weil die Konkretisierung des Sozialstaatsgrundsatzes tatsächlich nicht aus einer einheitlichen, sondern aus unterschiedlichen, allerdings durch Mehrheitsentscheidungen des Parlaments zu legitimierenden Sozialphilosophien hervorgeht, wird jedoch verkannt, dass auch (wenn auch nicht ausschließlich) Hellers Ansatz durch sozialdemokratische Vertreter im Parlamentarischen Rat im Grundgesetz seinen Niederschlag gefunden hat. Indem Gröschner den Sozialstaatsgrundsatz zu einem abstrakt allgemeinen Programm macht und durch die Verzeichnung der Entstehungsgeschichte um sein seinerzeitiges sozialdemokratisches Moment verkürzt, das auf die verfassungsrechtliche Absicherung der Möglichkeit einer Ausweitung demokratischer Teilhabe zielt, wird ein tragendes Element des Begriffs des sozialen Rechtsstaats zum Verschwinden gebracht.

Die dominierende und verkürzte Interpretation des Sozialstaatsgrundsatzes findet schon seit längerer Zeit in weitem Maße ihre Entsprechung in der Politik der Bundesregierung. Am Beispiel der Politik der Regierung Schröder/Fischer lässt sich zeigen, dass - trotz gewisser Erweiterungen der Mitbestimmung und staatlicher Programme zur Überwindung von Arbeitslosigkeit - jenes zentrale Mo-

I 3 Roman Herzog, in: Maunz-Dürig u.a., Grundgesetzkommentar, München I98 I, Art. 20, Rdnr. 47.

I4 Vgl. Rosa Luxemburg, Die russische Revolution (1918), Frankfurt/M 1963; Wolfgang Abendroth, Demokratie als Institution und Aufgabe (1954), in: ders., Arbeiterklasse, Staat und Verfassung, hrsg. v. Joachim Perels, Frankfurt/M i975, S. 2 I ff.

Is Vgl. Joachim Perels, Meinungsfreiheit als Element des Sozialismus (1979), in: ders., Demokratie und soziale Emanzipation, Hamburg 1988, S. I6 I ff; Lelio Basso, Die Rolle des Rechts in der Phase des Übergangs zum Sozialismus, Kritische Justiz, Heft 3/ı 973 , S. 239 ff.

I6 Rolf Gröschner, in: Dreier, Grundgesetz Kommentar, Tübingen I998, Art. 20, Rdnr. 9. 
ment des sozialen Rechtsstaats, das auf die Erweiterung der Bestimmungsmöglichkeiten der abhängig Arbeitenden im gesellschaftlichen Prozess zielt, keine strukturelle Bedeutung hatte. Am Beispiel der Arbeitsmarktpolitik, der Steuerpolitik und der europäischen Verfassungspolitik lässt sich dies zeigen: Durch die Privatisierung gesellschaftlich erzeugter Risiken, durch die massive Steuerentlastung von Großeigentümern und durch die Festschreibung der privaten Wirtschaft im europäischen Verfassungskonvent verliert der Grundsatz des sozialen Rechtsstaats an Bedeutung. Die vom Grundgesetz vorgegebene staatliche Verantwortung für die Überwindung struktureller sozialer Abhängigkeiten wurde eingeschränkt und durch die Sicherung partikularer Nutzungsmaximierung ersetzt.

Die mit der Agenda 20 Io verbundene Arbeitsmarktpolitik steht und fällt mit der Prämisse, dass die Arbeitslosigkeit durch individuelles Versagen begründet ist. Daher werden keine dem Sozialstaatsgedanken entsprechenden Konzeptionen entwickelt, in denen die individuellen Notlagen - von der Arbeitslosigkeit bis zur Krankheit - als gesellschaftliches Problem begriffen werden. Friedhelm Hengstbach hat dies eingehend herausgearbeitet: »Die Agenda 2010 bewirkt eine Deformation der Solidarität ... Strukturbrüche werden so dargestellt, als seien sie durch persönliches Fehlverhalten verursacht. Mangelnder Arbeitswille, geringe Qualifikation ..., ein leichtsinniger Charakter oder ein riskanter Lebensstil sind angeblich für das Eintreten gesellschaftlicher Risiken ... verantwortlich. Ein solches Deutungsmuster liefert ... den Vorwand dafür, die Bewältigung der (ökonomischen) Risiken der eigenen Initiative zu überlassen. Zwar reichen für den wohlhabenden Teil der Bevölkerung die Einkommen und Vermögen aus, um sich gegen die Lebensrisiken privat und kapitalgedeckt abzusichern, nicht jedoch für diejenigen, die bisher auf die solidarische umlagenfinanzierte Sicherung angewiesen waren. «17

Das Instrument, mit dem die abhängig Beschäftigten in die so genannte Eigenverantwortung gezwungen werden, besteht vor allem darin, dass die paritätische, von Arbeitgebern und Arbeitnehmern getragene Finanzierung der sozialen Sicherungssysteme für die Renten und das Krankengeld zur Entlastung der Unternehmer aufgehoben worden ist - mit der Folge, dass in der Versicherungswirtschaft möglicherweise ein Anlagenboom ausgelöst wird, während die Versicherten durch die Bereitstellung der entsprechenden Geldmittel Einschränkungen in ihrem Haushalt zu gewärtigen haben. Schließlich werden durch die Leistungskürzungen der Hartz IV-Gesetzgebung die sozialstaatlichen Auffangsysteme für Arbeitslose so verändert, dass frühzeitig für viele Menschen der Abstieg in die Armut - wie für 500.000 Kinder - programmiert ist, weil, um nur eine Zahl zu nennen, das Arbeitslosengeld, das bis zu 32 Monaten gezahlt wurde, für Beschäftigte unter 53 Jahren auf maximal I 2 Monate begrenzt wird. ${ }^{18}$ So werden diese Menschen auf sich selbst zurückgeworfen, unzureichend gestützt von der Sozialhilfe. Inzwischen wird vom Finanzminister der Regierung Merkel die Politik der Einschränkung und schließlichen Aufgabe des sozialstaatlichen Schutzes gegenüber bestimmten Gruppen offensiv propagiert. In einer programmatischen Rede vom I I. Januar 2006 stimmte Peer Steinbrück in aller Offenheit einem Abgesang auf jenes Element des demokratischen Rechtsstaats an, das ein soziales Minimum für die unmittelbaren Opfer der ökonomischen Krisenent- 
wicklung sichert: »Was aktivierend wirkt, muss bleiben und es kann sogar ausgebaut werden, wenn zugleich alles abgebaut wird, was zur Passivität und übertriebenen Anspruchshaltung führt. Es kann nicht das alleinige Ziel des modernen Staats sein, jeden Einzelnen gegen die Unwägbarkeiten des Marktes zu schützen. «19

Parallel zur Überwälzung gesellschaftlich erzeugter Problemlagen auf die große Masse der Einzelnen werden diejenigen zahlenmäßig kleinen Gruppen, die als Eigentümer und als Manager über den Inhalt und die Richtung des Produktionsprozesses bestimmen, durch die Steuerpolitik massiv privilegiert. Heribert Prantl hat dies konkret beschrieben: »Beim Regierungsantritt von Rot-Grün im Jahr 1998 hatten (die Körperschaftssteuer und die Einkommensteuer) noch einen Anteil (am Gesamtsteueraufkommen) von 6,7 Prozent. Im Jahr 200I nach drei Jahren rot-grüner Regierung, betrug der Anteil zusammen noch I,8 Prozent ... Im Jahr 200 I hat der Staat der Wirtschaft gar 426 Millionen Euro mehr an Körperschaftssteuer zurückerstattet als eingenommen. ${ }^{20}$ Die Steuerpolitik privilegiert die oberen Schichten gegenüber den lohnabhängigen Schichten und trägt dazu bei, dass - in der Periode der Massenarbeitslosigkeit - ı० Prozent aller Haushalte ihren Anteil am gesamten Geldvermögen von 44,5 Prozent im Jahr I 993 auf 5 I Prozent im Jahr 2003 steigern konnten. Die Kehrseite ist, dass die Steuerquote des Staats, von deren Höhe die Finanzierungsmöglichkeit der Sozialversicherungssysteme auch abhängt - etwa bei der Sicherung der Liquidität der Rentenkasse - zwischen 2000 und 2004, als Hans Eichel Finanzminister war, um I 3 Prozent fiel. Damit hat Deutschland die niedrigste Steuerquote der alten EULänder. Diese Grundtatsache, die Finanzminister Steinbrück in seiner schon erwähnten Rede ausdrücklich zur guten Nachricht erklärte, ist aber nichts anderes als die ökonomische Unterminierung des dem Sozialstaatsgedanken verpflichteten Aufgabenfeldes des Staats, das nicht nur die sozialen Sicherungssysteme, sondern auch die Verbesserung der Qualität von Krankenhäusern, die Humanisierung von Gefängnissen und die Unterstützung kultureller Tätigkeit einschließt. So führt die niedrige Steuerquote in Deutschland als Ergebnis der Politik der rot-grünen Koalition zu einer weitgehenden Abkehr vom Gedanken des sozialen Rechtsstaats. Dessen Realisierungsmöglichkeit erweist sich freilich auch unter kapitalistischen Bedingungen darin, dass in skandinavischen Ländern wie Dänemark und Schweden die Staatseinnahmen fast 20 Prozent höher liegen als in Deutschland und damit andere Ressourcen für die Bewältigung sozialer Probleme zur Verfügung stehen, ohne dass damit die wirtschaftliche Entwicklung entsprechend der weit verbreiteten These, dass staatliche Sozialleistungen eine Investitionsbremse seien, beeinträchtigt wurde. ${ }^{21}$

In der europäischen Verfassungspolitik schließlich, die von deutscher Seite wesentlich von Außenminister Fischer im Verfassungskonvent betrieben wurde, ist die Gedankenwelt des sozialen Rechtsstaats in dem vorgelegten und verabschiedeten Verfassungstext, der in rechtsstaatlicher Hinsicht etwa im Blick auf die Ahndung von Staatsverbrechen oder dem Schutz der Privatsphäre beachtliche Fortschritte bringt, weitgehend nicht berücksichtigt worden. Die soziale Sphäre, für die eine ganze Reihe von Schutzgarantien für die in abhängigen Beschäftigungsverhältnissen Tätigen festgelegt werden (Art. II-27, II-34), wird als privatwirtschaftliche Ordnung verfassungsrechtlich festgeschrieben. Sie bil-

I9 Rede des Bundesministers der Finanzen, Peer Steinbrück, am I .. I.2006, Internet-Ausdruck.

2I Kirchlicher Herausgeberkreis Gerechtigkeit, Armes reiches Deutschland, Frankfurt/Main 2005, S. I $98 \mathrm{ff}$. 
det den nicht einmal durch Staatszielbestimmungen begrenzten Inhalt der genuin bürgerlichen Verfassung. In der Präambel zur Charta der Grundrechte, die in die Verfassung inkorporiert worden sind, werden als Verfassungsprinzipien Rechtsstaatlichkeit und Demokratie genannt, während der Gedanke des sozialen Rechtsstaats, der sich nicht nur im Grundgesetz, sondern auch in Nachkriegsverfassungen Frankreichs und Italien findet, ${ }^{22}$ gar nicht erwähnt wird. Für die europäische Verfassung ist er kein Leitprinzip. Die Nichtrezeption dieser Verfassungstradition, auch durch die rot-grüne Regierung, hat eine bemerkenswerte Konsequenz: Die Unternehmerfreiheit, die in der Eigentumsgarantie des Grundgesetzes dem Vorbehalt der Inhaltsbestimmung durch die demokratischen Vertretungskörperschaften unterliegt, wird ohne jeden Vorbehalt als Grundrecht anerkannt (Art. II-I6). Mit dieser Festlegung wird der Rechtsgrundsatz des sozialen Rechtsstaats negiert und die existierende Wirtschaftsordnung zum alternativlosen Gesellschaftssystem hypostasiert.

Angesichts der umfassenden politischen Tendenz, die Prinzipien des sozialen Rechtsstaats den Reproduktionsbedingungen des gegenwärtigen Kapitalismus trotz gewisser Einschränkungen nicht nur unterzuordnen, sondern in wesentlichem Maße zu opfern, stellt sich die Frage nach der Möglichkeit einer Gegenstrategie, die ich freilich nur punktuell beantworten kann. Die Schwierigkeit, an Abendroths Sozialstaatsinterpretation unter heutigen Bedingungen anzuknüpfen, besteht darin, dass die Interpretationsherrschaft über die gesellschaftlichen Probleme der Bundesrepublik, insbesondere im Blick auf die Frage nach den Ursachen der Massenarbeitslosigkeit, in den Wirtschaftsteilen der großen Zeitungen und Zeitschriften - von der Frankfurter Allgemeinen Zeitung über die Süddeutsche Zeitung, die Welt, die Zeit bis zum Spiegel - ganz überwiegend in den Händen derjenigen liegt, die die wirtschaftliche Krise in den Kategorien der von den Unternehmern und von den meisten Politikern vertretenen Angebotslehre darstellen, der zufolge durch den Abbau des Sozialstaats eine investitionsfördernde Kostenminderung eintrete. Diese Generalthese ist in der Wirtschaftswissenschaft, aber auch in der Rechtswissenschaft dominant. Im Sachverständigenrat zur Begutachtung der gesamtwirtschaftlichen Entwicklung haben die Vertreter der Angebotslehre eine erdrückende Mehrheit. Im Jahr 2004 fand eine Tagung der Vereinigung Deutscher Staatsrechtslehrer statt, die Probleme des Sozialstaats zum Gegenstand hatte. Einer der Hauptreferenten, der Leipziger Öffentlichrechtler Professor Enders, hat in seinem Beitrag eingehend die Position von Ernst Forsthoff aus dem Jahr 1953 rezipiert und den Primat des bürgerlichen Rechtsstaats gegenüber dem Sozialstaat, der eine bloße Ergänzungsfunktion habe - als gäbe es diese Abstufung im Grundgesetz - behauptet. In dem Referat wird die Position Abendroths mit keinem Wort erwähnt. ${ }^{23}$ Dabei hatte Abendroth in seinem langen Diskussionsbeitrag, der sich direkt auf Forsthoff bezieht, dessen Infragestellung des Gedankens des sozialen Rechtsstaats kritisiert; in den Veröffentlichungen der Vereinigung Deutscher Staatsrechtslehrer von 1954 ist die gesamte Stellungnahme Abendroths abgedruckt. ${ }^{24}$ Durch Verschweigen kann Denken in Alternativen zum Status quo auch entsorgt werden.

22 Joachim Perels, Die historischen Wurzeln der europäischen Einigung und die gegenwärtige Konstituierung der Verfassung, in: Jutta Joachim u. a. (Hrsg.), Konstitutionalisierung und Governments in der EU - Perspektiven einer europäischen Verfassung, Münster 2006, S. $22 \mathrm{ff.}$

23 Christoph Enders, Sozialstaatlichkeit im Spannungsfeld von Eigenverantwortung und Fürsorge, Veröffentlichungen der Vereinigung der Deutschen Staatsrechtslehrer, Bd. 64, Berlin 2005, S. 9 ff., S. 49.

24 Veröffentlichungen der Vereinigung der Deutschen Staatsrechtslehrer, Bd. I 2, Berlin I954, S. 85 ff. 
Verstärkt wird die Affirmation der privaten Wirtschaftsordnung durch Gerhard Schröder, der in seiner Zeit als Bundeskanzler den Kapitalismus umfassend legitimierte. Ausdrücklich erklärte er: »Kein anderes System hat den Menschen mehr Freiheit, mehr Sicherheit und mehr Wohlstand bieten können. «25 Mit dieser - durchaus undifferenzierten - Bemerkung übersieht Schröder, dass in vielen Perioden kapitalistischer Herrschaft Freiheit und Sicherheit von Millionen Menschen zum Zweck der Systemerhaltung eingeschränkt und zerstört wurden: von der Zerschlagung der Arbeiterorganisationen und der systematischen Verfolgung und Ermordung ihrer Anhänger im privatwirtschaftlich strukturierten Nationalsozialismus über die Aushöhlung der Existenzgrundlagen der armen Bevölkerungsschichten der Dritten Welt durch die neoliberale Weltwirtschaftspolitik des Internationalen Währungsfonds und der Weltbank bis zur stagnierenden Massenarbeitslosigkeit in der Bundesrepublik, die die Lebensperspektiven vieler Menschen, unter dem Bann ungezügelter Marktgesetze, einengt und auch beseitigt. Dass ein (inzwischen ehemaliger) SPD-Bundeskanzler ein Meinungsführer des gegenwärtigen Kapitalismus ist, zeigt, auf welche Widerstände der ursprüngliche Gedanke des sozialen Rechtsstaats inzwischen stößt. Der kürzlich neu gewählte SPD-Vorsitzende Kurt Beck spricht, im Unterschied zu Schröder, mittlerweile davon, seine Partei müsse sich auf ihre Wurzeln besinnen, um im gleichen Atemzug die Orientierung an der neoliberalen Angebotslehre fortzusetzen. Er fordert die Senkung der Unternehmenssteuern und stellt gleichzeitig die vielfache Abwälzung von Sozialausgaben auf die Schlechtergestellten nicht in Frage. Für Willy Brandt, dem langjährigen Vorsitzenden der SPD in der Bundesrepublik, blieb die Gedankenwelt des sozialen Rechtsstaats verpflichtend. Programmatisch forderte er, aktuell bis heute, »dass die vielen und nicht nur die wenigen am Tisch der Gesellschaft Platz haben müssen. $\ll^{26}$

Ein bekanntes Wort von Marx lautet, dass nur dann, wenn die Wirklichkeit sich zum Gedanken drängt, aussichtsreich gehandelt werden kann. Der Satz von Marx muss heute umgekehrt werden. Gerade weil jedenfalls die Haupttendenzen der Wirklichkeit nicht zum Gedanken des sozialen Rechtsstaats drängen, muss der Versuch unternommen werden, die politische und ideologische Hegemonie der Kopflanger der gegenwärtigen Stufe des Kapitalismus in einem längeren Prozess zurückzudrängen, in dem auch die soziale Organisationsmacht der Gewerkschaften, die existentiell an die Verteidigung des Sozialstaatsprinzips gebunden sind, eine wichtige Rolle spielt. Heinrich Heine hat hierfür ein Stichwort gegeben. Er sagt, dass der kritische Gedanke, der die Wirklichkeit überspringt, der Tat so vorausgeht wie der Blitz dem Donner. 